\title{
APPROXIMATION OF THE SPHERE BY POLYTOPES HAVING FEW VERTICES
}

\author{
I. BÁRÁNY AND Z. FÜREDI
}

(Communicated by Andrew Odlyzko)

\begin{abstract}
How well can a polytope with $n$ vertices approximate the unit ball $B^{d}$ of the $d$-dimensional Euclidean space? The answer is quite well known when $d$ is fixed and $n$ tends to infinity. In this paper the same question is answered when $n$ is a function of $d$ (a polynomial in $d$, say) and $d$ tends to infinity. Some applications of the results are also indicated.
\end{abstract}

1. Introduction. Approximation of convex bodies by polytopes is a wellstudied subject in convexity theory. There are several exact results in the 2- and 3-dimensional space (see Fejes-Tóth [7]). For convex bodies in $R^{d}$ with $d>3$ most results are asymptotic. To give some examples let $\delta^{H}(C, P)$ denote the Hausdorff distance of $C, P \subset R^{d}$, and for a convex body $C \subset R^{d}$ define

$$
\delta^{H}(C, n)=\inf \left\{\delta^{H}(C, P): P \subset C \text { a polytope with } n \text { vertices }\right\} .
$$

It is known that for a convex body $C$ of class $C^{2}$

$$
\frac{\text { const }}{n^{2 /(d-1)}} \leq \delta^{H}(C, n) \leq \frac{\text { const }}{n^{2 /(d-1)}}, \quad n=d+1, d+2, \ldots
$$

The upper bound is due to Dudley [5], the lower bound is due to Schneider and Wieacker [13]. Schneider [12] determined the asymptotic behavior of $\delta^{H}(C, n)$ when $C$ is of class $C^{3}$ with positive Gaussian curvature:

$$
\lim _{n \rightarrow \infty} \delta^{H}(C, n)=\frac{\text { const }}{n^{2 /(d-1)}}
$$

where the constant depends on the curvature only. Another measure of approximation is the Lebesgue-measure. Let $\delta^{L}(C, P)$ be the volume of $(C \backslash P) \cup(P \backslash C)$, and for a convex body $C \subset R^{d}$ define

$$
\delta^{L}(C, n)=\inf \left\{\delta^{L}(C, P): P \subset C \text { a polytope with } n \text { vertices }\right\} .
$$

It follows from (1) and from a result of Gruber and Kendarov [9] that if $C$ is of class $C^{2}$ then

$$
\frac{\text { const }}{n^{2 /(d-1)}} \leq \delta^{L}(C, n) \leq \frac{\text { const }}{n^{2 /(d-1)}}, \quad n=d+1, d+2, \ldots .
$$

For further information on approximation see the excellent survey paper by Gruber [8]. We mention one more result: Macbeath [11] proved that among all convex bodies $C \subset R^{d}$ of equal volume $\delta^{L}(C, n)$ is maximal if and only if $C$ is an ellipsoid.

Received by the editors August 28, 1986 and, in revised form, November 4, 1986. $26 \mathrm{~B} 25$.

1980 Mathematics Subject Classification (1985 Revision). Primary 52A22, 52A25; Secondary 
In this paper the behavior of $\delta^{L}(C, n)$ will be investigated when $C=B^{d}$, the Euclidean unit ball of $R^{d}$, and $n$ is a function of $d$ (a polynomial in $d$, for instance) and $d$ tends to infinity. It turns out that $\delta^{L}\left(B^{d}, n\right)$ is very close to $\operatorname{vol}\left(B^{d}\right)$. Therefore it will be convenient to introduce

$$
V(d, n)=\frac{\max \left\{\operatorname{vol}\left(\operatorname{conv}\left\{x_{1}, \ldots, x_{n}\right\}\right): x_{1}, \ldots, x_{n} \in B^{d}\right\}}{\operatorname{vol}\left(B^{d}\right)}
$$

and

$$
W(d, n)=[V(d, n)]^{1 / d} .
$$

Thus $1-V(d, n)=\omega_{d}^{-1} \delta^{L}\left(B^{d}, n\right)$ where

$$
\omega_{d}=\operatorname{vol}\left(B^{d}\right)=\frac{\pi^{d / 2}}{\Gamma(d / 2+1)}
$$

2. Upper and lower bounds for $W(d, n)$. When $n=f(d)$ is a function of $d$ and $d$ tends to infinity the behavior of $W(d, n)$ can be determined using the theorems of the next section. Specializing to the cases when $n$ is linear, polynomial and exponential function of $d$ we obtain the following upper bounds:

$$
\begin{gathered}
W(d, c d)<\left(\frac{3 e \log c}{d}\right)^{1 / 2}(1+o(1)) \quad \text { when } c>1, \\
W\left(d, d^{a}\right)<\left(\frac{2 e(a-1) \log d}{d}\right)^{1 / 2}(1+o(1)) \quad \text { when } a>1, \\
W\left(d, c^{d}\right)<(2 e \log c)^{1 / 2}(1+o(1)) \quad \text { when } 1<c<1.052 \ldots, \\
W\left(d, c^{d}\right)<\frac{1}{2} c \quad \text { when } 1.052 \ldots \leq c \leq \sqrt{2} \\
W\left(d, c^{d-1}\right)<\left(1-c^{-2}\right)^{1 / 2} \quad \text { when } \sqrt{2}<c .
\end{gathered}
$$

(Here (5) and (6) both hold for all $1<c \leq \sqrt{2}$ but for smaller values (5) gives a better upper estimate.)

We will also prove the following lower bounds for $W(d, n)$ when $n=f(d)$ and $d$ tends to infinity:

$$
\begin{gathered}
W(d, c d)>\left(\frac{\log c}{2 d}\right)^{1 / 2}(1+o(1)) \quad \text { when } c>100 \text { (say) } \\
W\left(d, d^{\alpha}\right)>\left(\frac{2(a-1) \log d}{d}\right)^{1 / 2}(1+o(1)) \quad \text { when } a>1 \\
W\left(d, c^{d-1}\right)>\left(1-c^{-2}\right)^{1 / 2}(1+o(1)) \quad \text { when } c>1
\end{gathered}
$$

The asymptotic estimation given in (2) can be reformulated as

$$
1-c_{1} n^{-2 /(d-1)}<V(d, n)<1-c_{2} n^{-2 /(d-1)} .
$$

It will follow from our results that $V(d, n)$ is separated from 1 unless $n$ is larger than $d^{d / 2}$. More precisely we have that for $\alpha>0$

$$
V\left(d, d^{\alpha(d-1)}\right)<e^{-d^{1-2 \alpha} / 2}
$$

This shows that $V$ may be close to one only if $\alpha>\frac{1}{2}+t(d) / \log d$ where $t(d) \rightarrow \infty$ as $d \rightarrow \infty$. On the other hand we will give a simple construction showing that $V(d, n)$ 
is indeed close to one when $n=d^{\alpha(d-1)}$ and $\alpha>\frac{1}{2}+t(d) / \log d$ with $\lim t(d)=\infty$. More precisely, we will show that for $\alpha>0$

$$
V\left(d, d^{\alpha(d-1)}\right)>e^{-d^{1-2 \alpha}} .
$$

The proof of these results involves a lot of calculations. We will leave out most of the routine steps.

3. The theorems. Now we give the theorems that imply the lower and upper bounds of the previous section. The first result is due to Elekes [6]:

ThEOREM 1 (ElEKeS [6]). For $d \geq 2$ and $n \geq d+1$

$$
V(d, n) \leq n 2^{-d} \text {. }
$$

To present the second theorem define $M_{r}=\left(x+\frac{1}{2} B^{d}\right) \backslash r B^{d}$ where $x \in R^{d}$ with $\|x\|=\frac{1}{2}$.

THEOREM 2. For $d \geq 2, n \geq d+1$ and $1 / \sqrt{2}<r \leq 1$

$$
V(d, n) \leq r^{d}+n \omega_{d}^{-1} \operatorname{vol}\left(M_{r}\right) \text {. }
$$

THEOREM 3. For $d \geq 2, n \geq d+1$ and $k \in\{1,2, \ldots, d-1\}$

$$
V(d, n) \leq\left(\begin{array}{c}
n \\
k+1
\end{array}\right) \frac{\sqrt{k+1}}{k !}\left(1+\frac{1}{k}\right)^{k / 2} \frac{\omega_{d-k}}{\omega_{d}}\left(\frac{d-k}{d k}\right)^{(d-k) / 2}
$$

We will give lower bounds for $V(d, n)$. All but one of them come from a random construction. Unfortunately, there is no known formula for the expected volume of the convex hull of $n$ points chosen from $B^{d}$ (or $S^{d-1}$, the unit sphere) uniformly and independently. We use an integral formula due to Buchta, Müller and Tichy [3] which gives the expected surface area $E(d, n)$ of the convex hull of $n$ points chosen uniformly and independently from $S^{d-1}$ :

$$
\begin{aligned}
E(d, n)= & \left(\begin{array}{l}
n \\
d
\end{array}\right) \frac{d \gamma_{d-1}}{(d-1)^{d-1}}\left(\frac{\gamma_{d-1}}{\gamma_{d}}\right)^{d-1} \\
& \times \int_{-1}^{1}\left(\frac{\gamma_{d-1}}{\gamma_{d}} \int_{p}^{1}\left(1-q^{2}\right)^{(d-3) / 2} d q\right)^{n-d}\left(1-p^{2}\right)^{\left(d^{2}-d-2\right) / 2} d q
\end{aligned}
$$

where $\gamma_{d}=$ Area $S^{d-1}$ denotes the surface area of $S^{d-1}$.

THEOREM 4. For $d \geq 2$ and $n \geq d+1$

$$
V(d, n) \geq d^{-2} \omega_{d}^{-1} E(d, n-d) .
$$

The other lower bound shows that Elekes' estimation on $V(d, n)$ is quite good when $n$ is about $2^{d / 2}$.

THEOREM 5. When $n=20 d^{3 / 4} 2^{d / 2}$ and $d \geq 100$, then

$$
V(d, n) \geq \frac{1}{500 d^{3 / 2}} \cdot n 2^{-d} \text {. }
$$

We will not prove this theorem here because its proof is given in a companion paper [2]. 
4. Two related results and the Hausdorff measure. In the companion paper [2] the following problem is considered. Let $E_{n}$ denote the convex hull of $n$ points chosen uniformly and independently from $B^{d} . E_{n}$ is a convex polytope with at most $n$ vertices. It is proved in [2] that for $n=c 2^{d / 2}$ (with $c>0$ )

$$
\operatorname{Prob}\left(E_{n} \text { has exactly } n \text { vertices }\right)>1-c^{2},
$$

and for $n=c d^{3 / 4} 2^{d / 2}$ (with $c \geq 20$ and $d \geq 100$ )

$$
\operatorname{Prob}\left(E_{n} \text { has exactly } n \text { vertices }\right)<2 e^{-c / 2} \text {. }
$$

There we also consider the problem whether $E_{n}$ is or is not $k$-neighborly with probability tending to one as $d \rightarrow \infty$.

The small value of $W$ in the formulas (3)-(7) is somewhat surprising, at least for the authors. We mention that in another companion paper [1] we use (4) to show that in a certain model of computation the volume of convex bodies cannot be determined within a factor of $(d / \log d)^{d}$ by any polynomial-time algorithm. On the other hand Grötschel, Lovász and Schrijver [10] gave a polynomial-time algorithm which determines the volume of a centrally symmetric convex body within a factor of $d^{d}$. The interested reader is referred to [10 and $\mathbf{1}$.

It is perhaps interesting to note here that $\delta^{H}\left(B^{d}, n\right)$, the Hausdorff measure of the approximation, can be computed easily when $n$ is small (a polynomial in $d$, say). This is in contrast with the case when $d$ is fixed and $n$ tends to infinity, when, e.g., the upper bound in (1) implies the upper bound in (2). One way to compute $\delta^{H}\left(B^{d}, n\right)$ for $n=d^{d}$ is to show (by an averaging argument) that there is "big" spherical sector missing all the $n$ points. (Here sector means the convex hull of a spherical cap and the center.) Another way to compute $\delta^{H}\left(B^{d}, n\right)$ is to use (4) which says that the ball with radius $r=(1+o(1))(2 e(a-1) \log d / d)^{1 / 2}$ with center the origin is not contained in the approximating convex polytope. This implies that

$$
\delta^{H}\left(B^{d}, n\right)>1-(1+o(1))(2 e(a-1) \log d / d)^{1 / 2} .
$$

An easy random construction shows that this estimation is fairly good.

5. Two lemmas and an open question. The proofs of Theorems 1,2 and 3 are based on two lemmas. The first is due to Elekes [6]. For $z \in R^{d}$ define

$$
B(z)=\left\{x \in R^{d}:\left\|x-\frac{1}{2} z\right\| \leq \frac{1}{2}\|z\|\right\}
$$

that is, $B(z)$ is the ball with center $\frac{1}{2} z$ and radius $\frac{1}{2}\|z\|$.

LEMMA 1 (EleKeS [6]). For $d \geq 1, n \geq 1$, and $x_{1}, \ldots, x_{n} \in R^{u}$

$$
\operatorname{conv}\left\{x_{1}, \ldots, x_{n}\right\} \subseteq \bigcup_{i=1}^{n} B\left(x_{i}\right) .
$$

PrOOF. We reproduce Elekes' simple and elegant argument here. Consider $y \in \operatorname{conv}\left\{x_{1}, \ldots, x_{n}\right\}$. If $y=0$ then $y \in B\left(x_{i}\right)$ for every $i=1, \ldots, n$. So assume $y \neq 0$ and consider the closed halfspace

$$
H=\left\{x \in R^{d}:\langle x, y\rangle \geq\|y\|^{2}\right\} .
$$

As $y \in \operatorname{conv}\left\{x_{1}, \ldots, x_{n}\right\}$ there need be an index $i$ with $x_{i} \in H$. Then the angle of the triangle with vertices $0, x_{i}$ and $y$ at $y$ is at least $\pi / 2$. This shows that $y \in B\left(x_{i}\right)$. 
To formulate the second lemma we need some preparations. Assume $C \subset R^{d}$ is convex, compact. Denote by $L$ the orthogonal complementary subspace of aff $C$. For $\varsigma>0$ define the $\varsigma$-cylinder above $C$ as

$$
C^{\varsigma}=C+\left(L \cap \varsigma B^{d}\right)
$$

i.e. $C^{\varsigma}$ is the set of points $x \in R^{d}$ such that if $\bar{x}$ denotes the nearest point to $x$ in $C$ then $x-\bar{x}$ is orthogonal to aff $C$ and has length at most $\varsigma$. Finally define $\varsigma(d, 1)=1, \varsigma(d, d)=d^{-1}$ and

$$
\varsigma(d, k)=\left(\frac{d-k+1}{d(k-1)}\right)^{1 / 2} \text { for } k=2, \ldots, d-1 .
$$

LEMMA 2. If $X \subset B^{d}$ and $k \in\{1, \ldots, d\}$ then

$$
\operatorname{conv} X \subseteq \bigcup\left\{F^{\varsigma(d, k)}: F=\operatorname{conv} A\right\}
$$

where the union is taken over all $k$-tuples $A \subset X$.

REMARK. We conjecture that the smallest value of $\varsigma$ for which Lemma 2 holds is $((d-k+1) /(d k))^{1 / 2}$. This comes from the case when $|X|=d+1$ and conv $X$ is a regular simplex inscribed in $B^{d}$. For the time being we cannot prove this sharper version of Lemma 2. However, for our purposes the $\varsigma$ given in Lemma 2 will do and we cannot gain anything significant with the possibly best value of $\zeta$.

PROOF OF LEMMA 2. In view of Carathéodory's theorem [4] it is enough to prove the lemma when $|X|=d+1$, i.e., $F_{d+1}=\operatorname{conv} X$ is a simplex. Take $x \in F_{d+1}$.

We start with the case $k=1$ : a simple argument shows that $x$ is at distance one or less from some vertex of $F_{d+1}$. Consider next the case $k=d$. This is equivalent to the following well-known fact (see Fejes-Tóth [7], for instance). The ratio of the radii of the circumscribed and.inscribed balls of a simplex is at most $d$. This establishes the case $k=d$.

Now we prove the lemma for fixed $d$ by backward induction on $k$. Rename $x$ as $x_{d+1}$. By the above fact there is a facet $F_{d}$ of $F_{d+1}$ such that if $x_{d}$ denotes the orthogonal projection of $x_{d+1}$ onto aff $F_{d}$, then $\left\|x_{d+1}-x_{d}\right\| \leq d^{-1}$ and $x_{d+1}-x_{d}$ is orthogonal to aff $F_{d}=H_{d} . F_{d}$ lies in $B^{d} \cap H_{d}$ so it lies in $B^{d-1}$ as well if one chooses the origin in $H_{d}\left(\cong R^{d-1}\right)$ suitably. On applying the same argument to $F_{d} \subset B^{d-1}$ we get a point $x_{d-1}$ in a facet $F_{d-1}$ of $F_{d}$ such that $\left\|x_{d}-x_{d-1}\right\| \leq(d-1)^{-1}$ and $x_{d}-x_{d-1}$ is orthogonal to aff $F_{d-1}=H_{d-1}$. And so on. We stop with the $x_{k} \in F_{k}$. The vectors $x_{j+1}-x_{j}(j=d, \ldots, k)$ are pairwise orthogonal and all of them orthogonal to $F_{k}$. Consequently $x_{d+1}-x_{k}$ is orthogonal to $F_{k}$. Hence

$$
\begin{aligned}
\left\|x_{d+1}-x_{k}\right\|^{2} & =\sum_{j=d}^{k}\left\|x_{j+1}-x_{j}\right\|^{2} \leq \sum_{j=k}^{d} j^{-2} \\
& <\sum_{j=k}^{d}[j(j-1)]^{-1}=\sum_{j=k}^{d}\left(\frac{1}{j-1}-\frac{1}{j}\right)=\frac{d-k+1}{d(k-1)} .
\end{aligned}
$$

We think that there is a certain possibility to strengthen Lemma 2 in the following way. Let $X \subset R^{d}$ be a set of $d$ linearly independent vectors and consider a $k$-tuple $A \subset X$. Then there is a uniquely determined ellipsoid having the smallest $k$-dimensional volume among all ellipsoids lying in $\operatorname{lin} A$, the linear hull of $A$, and 
containing $A \cup\{0\}$. The center of this ellipsoid is $c(A)=(k+1)^{-1} \sum\{a: a \in A\}$. Denote by \|\|$_{A}$ the norm induced by this ellipsoid. Now take any point $x \in R^{d}$ and represent it as $x=x_{A}+x_{A}^{\mathbf{q}}$ where $x_{A} \in \operatorname{lin} A$ and $x_{A}^{\mathbf{q}} \in(\operatorname{lin} A)^{\Upsilon}$, the orthogonal complement of $\operatorname{lin} A$. Define another norm ||$_{A}$ with center $c(A)$ by

$$
|x-c(A)|_{A}^{2}=\frac{k+1}{k}\|x-c(A)\|_{A}^{2}+\mu^{2}(d, k)\left\|x_{A}^{\mathbf{q}}\right\|^{2}\left[\operatorname{vol}_{k}(\operatorname{conv}(A \cup\{0\}))\right]^{-2 / k} .
$$

The unit ball of this norm with center $c(A)$ is again an ellipsoid $E(A)$. Now the open question is this. What is the minimal value of $\mu(d, k)>0$ such that for every point $x \in \operatorname{conv}(X \cup\{0\})$ there is a $k$-tuple $A \subset X$ with $x \in E(A)$ ?

The case $k=1$ is the same as Elekes' lemma and we know that the smallest value of $\mu(d, 1)$ is 2 . The minimal value for $\mu(d, k)$ will probably come from the case when $X \subset S^{d-1}$ and conv $X$ is a regular $(d-1)$-dimensional simplex of suitable side length. With that value of $\mu(d, k)$ one can improve on the estimations (3), (4), (5).

6. Proof of Theorems $1,2,3$ and the upper bounds. Theorem $1 \mathrm{im}$ mediately follows from Lemma 1 . Theorem 2 follows from it as well because for $r>0$

$$
\bigcup_{i=1}^{n} B\left(x_{i}\right)=r B^{d} \cup \bigcup_{i=1}^{n}\left(B\left(x_{i}\right) \backslash\left(r B^{d}\right)\right),
$$

and so

$$
\operatorname{vol}\left(\operatorname{conv}\left\{x_{1}, \ldots, x_{n}\right\}\right) \leq r^{d} \operatorname{vol}\left(B^{d}\right)+n \operatorname{vol}\left(M_{r}\right),
$$

implying (14).

To prove Theorem 3 we use Lemma 2 . It implies

$$
\operatorname{vol}\left(\operatorname{conv}\left\{x_{1}, \ldots, x_{n}\right\}\right) \leq \sum \operatorname{vol}\left(\operatorname{conv}\left(F^{\varsigma}\right)\right)
$$

where the summation is taken over all $k$-dimensional simplices $F$ with vertices from $x_{1}, \ldots, x_{n}$. It is clear that

$$
\operatorname{vol}\left(\operatorname{conv}\left(F^{\varsigma}\right)\right)=\operatorname{vol}_{k}(\operatorname{conv}(F)) \cdot \operatorname{vol}_{d-k}\left(\varsigma B^{d-k}\right) .
$$

$F$ is a $k$-dimensional simplex lying in $B^{d}$ so its volume is maximal if it is regular. Now a simple computation proves Theorem 3 .

To prove the upper bounds we use Theorems 1, 2 and 3. (6) follows from Theorem 1 and (7) follows from Theorem 2 when we choose $r>0$ as to minimize the right-hand side of (14). In the given range this choice is

$$
r=\left(1-\left(\frac{\sqrt{\beta \pi d}}{n}\right)^{2 /(d-1)}\right)^{1 / 2}
$$

with $1 \leq \beta \leq 2$. This works when $n=c^{d-1}$ with $c>2^{-1 / 2}$ and, also, when $n=d^{\alpha(\overline{d-1})}$ proving (11).

The proofs of (3), (4) and (5) are similar. We choose $k \in\{1,2, \ldots, d-1\}$ so as to minimize the right-hand side of (15). When $n=c d, d^{a}$ and $c^{d}$, respectively, then the appropriate choice for $k$ is $d /(2 \log c), d /(2(a-1) \log d)$ and $1 /(2 e \log c)$, proving (3), (4) and (5). 
7. Proof of Theorem 4 and the lower bounds.

PROOF OF THEOREM 4. Choose $n-d$ points $x_{1}, \ldots, x_{n-d}$ from $S^{d-1}$ uniformly and independently. Take $d$ more points $z_{1}, \ldots, z_{d} \in S^{d-1}$ in such a way that $x_{1}, z_{1}, \ldots, z_{d}$ form the vertices of a regular simplex. Set

$$
C=\operatorname{conv}\left\{x_{1}, \ldots, x_{n-d}, z_{1}, \ldots, z_{d}\right\}, \quad C_{0}=\operatorname{conv}\left\{x_{1}, \ldots, x_{n-d}\right\}
$$

and denote by $L_{1}, \ldots, L_{m}$ the facets of $C$. As $C$ contains $d^{-1} B$ we have

$$
\operatorname{vol}(C) \geq d^{-2} \sum_{i=1}^{m} \operatorname{vol}_{d-1}\left(L_{i}\right)=d^{-2} \operatorname{Area}(C) .
$$

Moreover, $C \supset C_{0}$ implies $\operatorname{Area}(C) \geq \operatorname{Area}\left(C_{0}\right)$. This proves the theorem.

The lower bounds (8), (9) and (10) follow from Theorem 4 and the integral formula (16) in the following way. First compute the value $p=p_{\text {crit }}$ where the function under integration in (16) takes its maximal value. Then compute a small interval $\left(p_{\text {crit }}-\Delta, p_{\text {crit }}+\Delta\right)$ where the function is close to its maximum. Using this, estimate $E(d, n-d)$ from below and apply Theorem 4 .

We elaborate the case $n=d^{a}(a>1)$ in more detail. Note, first, that for $p<0$

$$
\begin{aligned}
\int_{-1}^{p}\left(1-q^{2}\right)^{(d-3) / 2} d q & \leq \int_{-1}^{p} \frac{|q|}{|p|}\left(1-q^{2}\right)^{(d-3) / 2} d q \\
& =\frac{\left(1-p^{2}\right)^{(d-1) / 2}}{|p|(d-1)} \leq \frac{e^{-p^{2}(d-1) / 2}}{|p|(d-1)} .
\end{aligned}
$$

Anticipating $p_{\text {crit }}=-\sqrt{x \log d} / d$ and using $\gamma_{d-1} / \gamma_{d} \approx \sqrt{d / 2 \pi}$ we get from here

$$
\begin{aligned}
\left(\frac{\gamma_{d-1}}{\gamma_{d}} \int_{p}^{1}\left(1-q^{2}\right)^{(d-3) / 2} d q\right)^{n-2 d} & \\
& \geq\left(1-\frac{\gamma_{d-1}}{\gamma_{d}} \int_{-1}^{p}\left(1-q^{2}\right)^{(d-3) / 2} d q\right)^{n} \\
& \geq\left(1-\frac{\gamma_{d-1}}{\gamma_{d}} \frac{e^{-p^{2}(d-1) / 2}}{|p|(d-1)}\right)^{n} \geq\left(1-\frac{d^{-x / 2}}{\sqrt{2 \pi x \log d}}\right)^{n} \\
& \geq \exp \left\{-(1+\varepsilon)\left[n d^{-x / 2}(2 \pi x \log d)^{-1 / 2}\right]\right\}
\end{aligned}
$$

where the last step is justified by the inequality

$$
1-y \geq e^{-(1+\varepsilon) y}
$$

which is valid for $0<y<2 \varepsilon /(1+\varepsilon)$ if $\varepsilon \in(0,1 / 2)$, say. Similarly

$$
\left(1-p^{2}\right)^{\left(d^{2}-d-2\right) / 2} \geq\left(1-p^{2}\right)^{d^{2} / 2} \geq \exp \left\{-(1+\varepsilon)\left[\frac{x d \log d}{2}\right]\right\} .
$$

This shows that, writing $f(p)$ for the integrand in (16) with $E(d, n-d)$ instead $E(d, n)$ and $p=p(x)=-\sqrt{x \log d} / d$,

$$
f(p(x)) \geq \exp \left[-\left\{(1+\varepsilon)\left[d^{a-x / 2}(2 \pi x \log d)^{-1 / 2}+\frac{x}{2} d \log d\right]\right\}\right] .
$$

Choose now

$$
x=x(z)=2(a-1)-\frac{\log \log d}{\log d}-\frac{\log 2 \pi(2(a-1)-\delta)}{\log d}-\frac{2 \log (1+z)}{\log d}
$$


where $z$ is close to zero and $\delta>0$ is small enough $2(a-1)-\delta>0$. Then from (20), and (22), (23)

$$
\begin{gathered}
f(p(x(z))) \geq \exp [-\{(1+\varepsilon) d[(1+z)-\log (1+z)+(a-1) \log d \\
\left.\left.\left.-\frac{1}{2} \log \log d-\frac{1}{2} \log (2 \pi(2(a-1)-\delta))\right]\right\}\right] \\
\geq \exp \left[-\left\{( 1 + \varepsilon ) d \left[1+z^{2} / 2+(a-1) \log d\right.\right.\right. \\
\left.\left.\left.-\frac{1}{2} \log \log d-\frac{1}{2} \log (2 \pi(2(a-1)-\delta))\right]\right\}\right] .
\end{gathered}
$$

This function is concave between $z_{1}=-1 / \sqrt{a}$ and $z_{2}=1 / \sqrt{a}$. Moreover,

$$
\left|p\left(z_{1}\right)-p\left(z_{2}\right)\right| \geq \sqrt{2 /(a-1) d \log d} .
$$

Now we see that (24) holds with $\varepsilon=a \log d / d$ when $y=p^{2}(x)$ from (22) and when $y=d^{-x / 2}(2 \pi x \log d)^{-1 / 2}$ from (20). So we have for $d$ large enough

$$
\begin{aligned}
\int_{-1}^{1} f(p) d p \geq\left|p\left(z_{1}\right)-p\left(z_{2}\right)\right| \min _{z_{1} \leq z \leq z_{2}} f(p(x(z))) \geq\left(\frac{2}{(a-1) d \log d}\right)^{1 / 2} \\
\times \exp [-d[1+(a-1) \log d \\
\left.\left.\quad-\frac{1}{2} \log \log d-\frac{1}{2} \log (2 \pi(2(a-1)-\delta))\right]\right] \exp \left\{-a^{2} \log ^{2} d\right\} .
\end{aligned}
$$

Using this in (16) and then in (17) we get the lower bound (9).

The proofs of (8) and (9) are quite similar and are, therefore, omitted.

Finally, to prove (12) we use the following well-known fact (see [7], e.g.). There is a set $X$ of $n$ points on $S^{d-1}$ such that the balls $x+\frac{1}{2} r B^{d}$ and $y+\frac{1}{2} r B^{d}$ are disjoint (for $x, y \in X, x \neq y$ ) but the balls $x+r B^{d}(x \in X)$ cover $S^{d-1}$ and $r \leq n^{-1 /(d-1)}$.

Then it can be seen that the inscribed ball of conv $X$ (with center the origin) has radius $R$ and

$$
\begin{gathered}
R \geq\left(1-r^{2}\right)^{1 / 2} \\
V(d, n) \geq R^{d} \geq\left(1-r^{2}\right)^{d / 2} \geq\left(1-n^{-2 /(d-1)}\right)^{d / 2} .
\end{gathered}
$$

When $\alpha>0$ and $n=d^{\alpha(d-1)}$ this gives (12).

\section{REFERENCES}

1. I. Bárány and Z. Füredi, Computing the volume is difficult, Discrete and Computational Geometry 2 (1987), 319-326 and Proc. 18th ACM STOC, 1986, pp. 442-447.

2.

3. C. Buchta, J. Müller and R. F. Tichy, Stochastical approximation of convex bodies, Math. Ann. 271 (1985), 225-235.

4. L. Danzer, B. Grünbaum and V. Klee, Helly's theorem and its relatives, Proc. Sympos. Pure Math., vol. 7, Amer. Math. Soc., Providence, R. I., 1963, pp. 101-180.

5. R. Dudley, Metric entropy of some classes of sets with differentiable boundaries, J. Approx. Theory 10 (1974), 227-236; Correction, ibid. 26 (1979), 192-193.

6. G. Elekes, $A$ geometric inequality and the complexity of computing the volume, Discrete and Computational Geometry 1 (1986), 289-292.

7. L. Fejes-Tóth, Regular figures, Pergamon Press, 1964.

8. P. M. Gruber, Approximation of convex bodies, Convexity and its Applications (P. M. Gruber and J. M. Wills, eds.), Birkhäuser, 1983, pp. 131-162. 
9. P. M. Gruber and P. Kendarov, Approximation of convex bodies by polytopes, Rend. Circ. Mat. Palermo 31 (1982), 195-225.

10. L. Lovász, An algorithmic theory of numbers, graphs and convexity, Report No. 85368-OR, Univ. Bonn, 1985.

11. A. M. Macbeath, An extremal property of the hypersphere, Proc. Cambridge Philos. Soc. 47 (1951), 245-247.

12. R. Schneider, Zur optimalen Approximation konvexer Hyperflächen durch Polyeder, Math. Ann. 256 (1981), 289-301.

13. R. Schneider and J. A. Wieacker, Approximation of convex bodies by polytopes, Bull. London Math. Soc. 13 (1981), 149-156.

DEPARTMENT OF MATHEMATICS, ORIE, UPSON HALL, CORNELl UNIVERSity, ITHACA, NEW YORK 14853

AT \& T Bell Laboratories, 2C-256, Murray Hill, NeW Jersey 07974

Permanent address of both authors: Mathematics Institute of the Hungarian Academy of Sciences, P. O. Box 127, 1364 Budapest, Hungary 